\title{
Radiative neutrino mass in 3-3-1 scheme
}

\author{
Sofiane M. Boucenna, ${ }^{1, *}$ Stefano Morisi, ${ }^{2, \dagger}$ and José W. F. Valle ${ }^{1, \sharp}$ \\ ${ }^{1}$ Instituto de Física Corpuscular (CSIC-Universitat de València), Apartado 22085, E-46071 Valencia, Spain \\ ${ }^{2}$ DESY, Platanenallee 6, D-15735 Zeuthen, Germany
}

(Received 13 May 2014; published 9 July 2014)

\begin{abstract}
We propose a new radiative mechanism for neutrino mass generation based on the $\mathrm{SU}(3)_{c} \otimes \mathrm{SU}(3)_{L} \otimes$ $\mathrm{U}(1)_{X}$ electroweak gauge group.Lepton number is a symmetry of the Yukawa sector which is spontaneously broken in the gauge sector. As a result light Majorana masses arise from neutral gauge boson exchanges at the one-loop level. In addition to the isosinglet neutrinos that may be produced at the LHC through the extended gauge boson portals, the model contains new quarks which can also lie at the $\mathrm{TeV}$ scale, and which can provide a plethora of accessible collider phenomena.
\end{abstract}

DOI: 10.1103/PhysRevD.90.013005

The origin of neutrino mass and mixing, which is required in order to account for neutrino oscillation data $[1,2]$, poses one of the biggest challenges in particle physics. While charged fermions must be Dirac particles, neutrinos are generally expected to be Majorana fermions [3], breaking the lepton number and inducing neutrinoless double-beta decay [4]. While attractive, the idea that neutrino mass is related to unification, encoded in the high-scale seesaw paradigm [3,5-8], falls short of covering the wealth of interesting neutrino mass schemes. Indeed neutrino masses could well be a low-scale phenomenon, both within the seesaw mechanism as well as in other alternative approaches [9]. This brings in substantial freedom in model building, making the structure of the leptonic weak interaction much richer than the CabibboKobayashi-Maskawa matrix [10,11] characterizing the quark sector, and opening the exciting possibility of probing the associated neutrino mass messenger particles at collider experiments [12-14].

Lepton number symmetry provides an important theoretical guide in neutrino mass modeling; depending on its fate, different classes of models can be envisaged. For instance, the lepton number can be conserved, leading to Dirac neutrinos. Or, it can be violated explicitly, since gauge singlet Majorana masses can be added by hand in the $\mathrm{SU}(3)_{c} \otimes \mathrm{SU}(2)_{L} \otimes \mathrm{U}(1)_{Y}$ model [3]. Or, it can be a spontaneously broken global or gauged U(1) symmetry. The former defines the so-called seesaw Majoron schemes $[15,16]$, while the latter characterizes left-right symmetric electroweak models [17]. Another important challenge is the origin of the number of families. We know that three different flavors exist, i.e., states with the same gauge quantum numbers but different mass. But we do not know why nature replicates, nor why the masses of the three generations of Standard Model (SM) quarks and leptons are

\footnotetext{
*boucenna@ific.uv.es

†stefano.morisi@gmail.com

‡valle@ific.uv.es
}

PACS numbers: $14.60 . \mathrm{Pq}, 12.60 . \mathrm{Cn}, 14.60 . \mathrm{St}, 14.70 . \mathrm{Pw}$

so different, nor why they mix in the way they do (the flavor problem).

In this paper we consider an alternative approach to neutrino mass generation at accessible scales and to "explaining" the number of families. The model is based on the $\mathrm{SU}(3)_{c} \otimes \mathrm{SU}(3)_{L} \otimes \mathrm{U}(1)_{X}$ (3-3-1) electroweak gauge structure, and it is consistent only if the number of families equals the number of quark colors [18,19]; this gives a reason for having three species of fermions. This feature follows from gauge anomaly cancellation and characterizes 3-3-1 models, including other variants, e.g., [20-25].

Our new mechanism for generating neutrino mass at the one-loop level involves a neutral gauge-mediated lepton number-violating interaction. All new particles, including the messengers associated to neutrino mass generation, can lie at the few-TeV scale, and, hence, are accessible to collider searches.

We start from the $\mathrm{SU}(3)_{c} \otimes \mathrm{SU}(3)_{L} \otimes U(1)_{X}$ gauge framework suggested in $[18,19]$. We concentrate on the electroweak part of the model. The left-handed leptons are assigned to the antitriplet representation of $S U(3)_{L}$,

$$
\psi_{L}^{\ell}=\left(\begin{array}{c}
\ell^{-} \\
\nu_{\ell} \\
N_{\ell}^{c}
\end{array}\right)_{L},
$$

where $\ell=1,2,3 \equiv e, \mu, \tau$. In addition to the new twocomponent neutral fermions present in the lepton triplet $N_{L}^{c} \equiv\left(N^{c}\right)_{L} \equiv\left(\nu_{R}\right)^{c}$, where $\psi^{c}=C \bar{\psi}^{T}$ and $C$ is the charge conjugation matrix, we introduce new sequential lepton number-carrying gauge singlets $S=\left\{S_{1}, S_{2}, S_{3}\right\}$ sequentially [26-30]. The matter content of the model is summarized in Table I.

With the above $\mathcal{L}$ assignment the electric charge and lepton number are given in terms of the $U(1)_{X}$ generator $X$ and the diagonal generators of the $S U(3)_{L}$ as 
TABLE I. Matter content of the model, where $\hat{u}_{R} \equiv$ $\left(u_{R}, c_{R}, t_{R}, t_{R}^{\prime}\right)$ and $\hat{d}_{R} \equiv\left(d_{R}, s_{R}, b_{R}, d_{R}^{\prime}, s_{R}^{\prime}\right)$ (see text).

\begin{tabular}{lcccccccccc}
\hline \hline & $\psi_{L}^{\ell}$ & $\ell_{R}$ & $Q_{L}^{1,2}$ & $Q_{L}^{3}$ & $\hat{u}_{R}$ & $\hat{d}_{R}$ & $S$ & $\phi_{1}$ & $\phi_{2}$ & $\phi_{3}$ \\
\hline$S U(3)_{c}$ & $\mathbf{1}$ & $\mathbf{1}$ & $\mathbf{3}$ & $\mathbf{3}$ & $\mathbf{3}$ & $\mathbf{3}$ & $\mathbf{1}$ & $\mathbf{1}$ & $\mathbf{1}$ & $\mathbf{1}$ \\
$S U(3)_{L}$ & $\mathbf{3}^{*}$ & $\mathbf{1}$ & $\mathbf{3}$ & $\mathbf{3}^{*}$ & $\mathbf{1}$ & $\mathbf{1}$ & $\mathbf{1}$ & $\mathbf{3}^{*}$ & $\mathbf{3}^{*}$ & $\mathbf{3}^{*}$ \\
$U(1)_{X}$ & $-\frac{1}{3}$ & -1 & 0 & $+\frac{1}{3}$ & $+\frac{2}{3}$ & $-\frac{1}{3}$ & 0 & $+\frac{2}{3}$ & $-\frac{1}{3}$ & $-\frac{1}{3}$ \\
$\mathcal{L}$ & $-\frac{1}{3}$ & -1 & $-\frac{2}{3}$ & $+\frac{2}{3}$ & 0 & 0 & 1 & $+\frac{2}{3}$ & $-\frac{4}{3}$ & $+\frac{2}{3}$ \\
\hline \hline
\end{tabular}

$$
\begin{gathered}
Q=T_{3}+\frac{1}{\sqrt{3}} T_{8}+X, \\
L=\frac{4}{\sqrt{3}} T_{8}+\mathcal{L} .
\end{gathered}
$$

In order to spontaneously break the weak gauge symmetry, we introduce three scalar antitriplets $\phi_{1} \sim$ $\left(\mathbf{3}^{*},+2 / 3\right)$ and $\phi_{2,3} \sim\left(\mathbf{3}^{*},-1 / 3\right)$. Note that the third component of $\phi_{3}$ carries two units of lepton number. Following the notation of [19] we have the following vacuum expectation values (VEVs):

$$
\left\langle\phi_{1}\right\rangle=\left[\left(\begin{array}{c}
k_{1} \\
0 \\
0
\end{array}\right)\right],\left\langle\phi_{2}\right\rangle=\left[\left(\begin{array}{c}
0 \\
0 \\
n_{1}
\end{array}\right)\right],\left\langle\phi_{3}\right\rangle=\left[\left(\begin{array}{c}
0 \\
k_{2} \\
n_{2}
\end{array}\right)\right],
$$

where the $k_{1}$ and $k_{2}$ VEVs are at the electroweak scale and correspond to the VEV of the $S U(2)_{L} \subset S U(3)_{L}$ doublets. The VEVs $n_{1}$ and $n_{2}$ are isosinglet VEVs that characterize the $S U(3)_{L}$ breaking scale. Note that while $\phi_{3}$ takes VEV in both electrically neutral directions, the second VEV of $\phi_{2}$ is neglected, so that lepton number is broken only by $S U(2)_{L}$ singlets. This pattern gives the simplest consistent neutrino mass spectrum, avoiding the linear seesaw contribution $[31,32]$.

The kinetic term for the scalar fields is

$$
\mathcal{L}_{\text {Kin }}=\sum_{i}\left(D^{\mu} \phi_{i}\right)^{\dagger}\left(D_{\mu} \phi_{i}\right)
$$

We define the covariant derivative as

$$
D_{\mu} \phi_{i}=\partial_{\mu} \phi_{i}+i \frac{g_{1}}{\sqrt{2}} \mathbf{W}_{\mu} \cdot \phi_{i}+i \sqrt{2} g_{2} X_{i} \mathbf{B}_{\mu} \phi_{i}
$$

where $X_{i}$ is the $U(1)_{X}$ charge of the $\phi_{i}$ scalar field, $\mathbf{B}_{\mu}$ is the gauge boson of $U(1)_{X}$, and $\mathbf{W}_{\mu} \equiv \sum_{i} W_{\mu}^{i} \lambda_{i}$ where $\lambda_{i}$ are the Gell-Mann matrices. In matrix form one has

$$
\mathbf{W}=\left(\begin{array}{ccc}
W^{3}+\frac{1}{\sqrt{3}} W^{8} & W_{12}^{+} & W_{45}^{+} \\
W_{12}^{-} & -W^{3}+\frac{1}{\sqrt{3}} W^{8} & W^{6}-i W^{7} \\
W_{45}^{-} & W^{6}+i W^{7} & -\frac{2}{\sqrt{3}} W^{8}
\end{array}\right),
$$

where for simplicity we dropped the index $\mu$ from the gauge bosons and defined the charged states as

$$
W_{12}^{ \pm}=\frac{1}{\sqrt{2}}\left(W^{1} \mp i W^{2}\right), \quad W_{45}^{ \pm}=\frac{1}{\sqrt{2}}\left(W^{4} \mp i W^{5}\right) .
$$

There are in total nine electroweak gauge bosons, four of which are charged $\left(W_{12}^{ \pm}\right.$and $\left.W_{45}^{ \pm}\right)$, giving the physical $W^{ \pm}$ and $W^{\prime \pm}$, while five are electrically neutral, namely $W^{3}, W^{6}$, $W^{8}$, and $B$, giving the physical bosons

$$
\gamma, \quad Z, \quad Z^{\prime}, \quad X
$$

and, finally, one neutral boson, unmixed if $C P$ is conserved,

$$
W^{7} \equiv Y .
$$

Assuming for simplicity $k_{2} \sim k_{1} \ll n_{1} \sim n_{2}$ one finds the gauge boson masses to leading order

$$
\begin{aligned}
m_{W}^{2} & =g_{1}^{2}\left(k_{1}^{2}+\frac{k_{2}^{2} n_{1}^{2}}{n_{1}^{2}+n_{2}^{2}}\right), \\
m_{W^{\prime}}^{2} & =g_{1}^{2}\left(n_{1}^{2}+n_{2}^{2}\right), \\
m_{Z}^{2} & =\frac{g_{1}^{2}\left(3 g_{1}^{2}+4 g_{2}^{2}\right)}{3 g_{1}^{2}+g_{2}^{2}}\left(k_{1}^{2}+\frac{k_{2}^{2} n_{1}^{2}}{n_{1}^{2}+n_{2}^{2}}\right), \\
m_{Z^{\prime}}^{2} & =\frac{4}{9}\left(3 g_{1}^{2}+g_{2}^{2}\right)\left(n_{1}^{2}+n_{2}^{2}\right), \\
m_{X}^{2} & =m_{Y}^{2}=g_{1}^{2}\left(n_{1}^{2}+n_{2}^{2}\right) .
\end{aligned}
$$

The ratio of the SM gauge boson masses is given by

$$
\frac{m_{W}^{2}}{m_{Z}^{2}}=\frac{3+\tan ^{2} \theta_{331}}{3+4 \tan ^{2} \theta_{331}},
$$

where $\tan ^{2} \theta_{331} \equiv g_{2} / g_{1}$. The SM relation $\cos ^{2} \theta_{W} \equiv$ $m_{W}^{2} / m_{Z}^{2}=0.76$ implies that $\tan ^{2} \theta_{331}=0.57$. The leptonic neutral-current weak interaction $\mathcal{L}_{\mathrm{NC}}$ contains

$$
\begin{aligned}
\mathcal{L}_{\mathrm{NC}} \supset & \frac{g_{1}}{\sqrt{2}} \overline{\nu_{L}} \gamma_{\mu} \nu_{L} W_{\mu}^{3}-\frac{g_{1}}{\sqrt{6}} \overline{\nu_{L}} \gamma_{\mu} \nu_{L} W_{\mu}^{8} \\
& -\frac{g_{1}}{\sqrt{2}} \overline{N_{L}^{c}} \gamma_{\mu} \nu_{L} W_{\mu}^{6}+\frac{\sqrt{2} g_{2}}{3} \overline{\nu_{L}} \gamma_{\mu} \nu_{L} B_{\mu} .
\end{aligned}
$$

The mixing of $W^{6}$ with $W^{3}, W^{8}$, and $B$ is proportional to the small parameter given by

$$
\epsilon \sim \frac{k_{2} n_{2}}{n_{1}^{2}+n_{2}^{2}} \ll 1 .
$$


RADIATIVE NEUTRINO MASS IN THE SU(3) ...

In addition to breaking the standard electroweak symmetry, this mixing also violates lepton number by two units, as can be readily seen through its proportionality to $n_{2}$, which is the scale of lepton number violation. The spontaneous symmetry breaking follows the pattern

$$
S U(3)_{L} \otimes U(1)_{X} \stackrel{n_{1,2}}{\rightarrow} S U(2)_{L} \otimes U(1)_{Y} \stackrel{k_{1,2}}{\rightarrow} U(1)_{Q} .
$$

Turning to the lepton sector, the Yukawa terms are

$$
\begin{aligned}
\mathcal{L}_{\text {leptons }}= & y_{i j}^{\ell} \overline{\psi_{L}^{i}} l_{R}^{j} \phi_{1}+y_{i j}^{a} \psi_{L}^{i T} C^{-1} \psi_{L}^{j} \phi_{1} \\
& +y_{i j}^{s} \overline{\psi_{L}^{i}} S^{j} \phi_{2}+\text { H.c. },
\end{aligned}
$$

where contraction of the flavor indices $i, j=1,2,3$ is assumed. Here $y^{\ell}$ and $y^{s}$ are arbitrary matrices while $y^{a}$ is antisymmetric. The charged lepton mass matrix is just $M_{\ell}=y^{\ell}\left\langle\phi_{1}^{0}\right\rangle$ and can be made diagonal in the usual way. Note that, thanks to an auxiliary parity symmetry, $\phi_{3}$ does not couple to leptons. The tree-level neutrino mass matrix in the basis $\left(\nu_{L}, N^{c}, S\right)$ is given by

$$
M_{\nu}=\left(\begin{array}{ccc}
0 & m_{D} & 0 \\
& 0 & M \\
& & 0
\end{array}\right),
$$

where $m_{D}=k_{1} y^{a}$ and $M=n_{1} y^{s}$. Note that lepton number conservation forbids the Majorana mass entry for $S$. We denote the corresponding eigenstates as $\nu_{1}, \nu_{2}, \nu_{3}$. The heavy states form Dirac pairs with masses $M_{D i}(i=1,2,3)$ given by

$$
M_{D i}=\left(\sqrt{m_{D} \cdot m_{D}^{T}+M \cdot M^{T}}\right)_{i}
$$

where the index $i$ in the r.h.s denotes the $i^{\text {th }}$ eigenvalue of the matrix [26-30]. On the other hand the state $\nu_{1}$ is massless because of lepton number conservation in Eq. (14). This holds at tree level. However, lepton number is broken spontaneously by $n_{2} \neq 0$. This induces light neutrino masses radiatively, as illustrated by the diagram in Fig. 1. In order to estimate the effective light neutrino mass scale that results from Eq. (15) we adopt, for simplicity, the one-family approximation (generalization to three is straightforward). Indeed, the interplay of the intramultiplet gauge boson exchange connecting $\nu$ to $N^{c}$ with the gauge boson mixing implicit in Eq. (13) implies that lepton number is necessarily violated in the neutral fermion sector. As a result the massless neutrino is not protected and radiative corrections involving the gauge bosons will yield a calculable Majorana mass term as depicted in the diagram of Fig. 1. To perform the corresponding estimate one goes to the mass basis. The result is that the light neutrino $\nu_{1}$ gets a Majorana mass by means of the exchange of the massive Dirac states $\nu_{2,3}$ and the gauge
PHYSICAL REVIEW D 90, 013005 (2014)

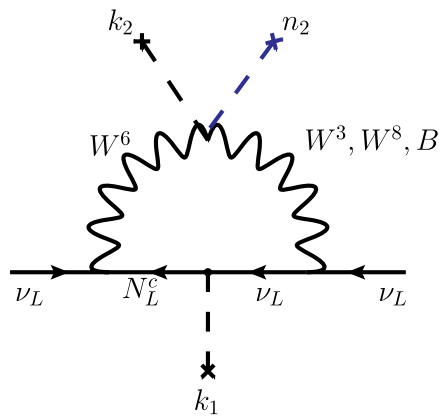

FIG. 1 (color online). Gauge boson exchange diagram for radiatively induced Majorana neutrino mass in the flavor basis.

bosons $Z$ and $Z^{\prime}$. Now we describe in more detail how this works.

Consider the $3 \times 3$ mass matrix given in Eq. (15). This matrix is diagonalized by an orthogonal matrix, given by a 1-3 rotation followed by a $2-3$ maximal rotation,

$$
U_{\nu}=\left(\begin{array}{ccc}
c & -\frac{s}{\sqrt{2}} & \frac{s}{\sqrt{2}} \\
0 & \frac{1}{\sqrt{2}} & \frac{1}{\sqrt{2}} \\
-s & -\frac{c}{\sqrt{2}} & \frac{c}{\sqrt{2}}
\end{array}\right),
$$

where $c \approx 1$ and $s \equiv \beta \simeq m_{D} / M \ll 1$ characterizes the doublet-singlet mixing, which could be at most a few percent due to universality constraints. Then the states $\nu_{L}$, $N_{L}^{c}, S$ are related to the massive neutrino states $\nu_{1}, \nu_{2}, \nu_{3}$ by (up to corrections of order $\epsilon$ )

$$
\begin{aligned}
\nu_{L} & \simeq \nu_{1}-\beta \nu_{2}+\beta \nu_{3}, \\
N_{L}^{c} & \simeq \frac{1}{\sqrt{2}}\left(\nu_{2}+\nu_{3}\right), \\
S & \simeq-\beta \nu_{1}+\frac{1}{\sqrt{2}}\left(-\nu_{2}+\nu_{3}\right) .
\end{aligned}
$$

Although both $Z$ and $Z^{\prime}$ enter in the loop, the main contribution is from $Z^{\prime}$ exchange, estimated from Eq. (18) and the diagonalization of the gauge bosons to be

$$
m_{\nu_{\text {light }}} \simeq \frac{g^{2} \epsilon \beta}{16 \pi^{2}} M_{D} \frac{m_{Z^{\prime}}^{2}}{M_{D}^{2}+m_{Z^{\prime}}^{2}} \log \frac{m_{Z^{\prime}}^{2}}{M_{D}^{2}}
$$

where $g$ is a simple function of gauge coupling constrants $g_{1}$ and $g_{2}$. Note that the contribution proportional to $\epsilon^{2}$ and $\beta^{2}$ vanish as expected.

Figure 2 shows the correlation between the light neutrino mass scale and the $Z^{\prime}$ mass for various values of the Dirac mass $M_{D}$, parametrized by the Yukawa coupling $y^{a}$. For definiteness we fix the $n_{2} \mathrm{VEV}$, responsible for the masses of the new isosinglet colored states, at $1 \mathrm{TeV}$ and $10 \mathrm{TeV}$. Increasing $n_{2}$ would push up the $Z^{\prime}$ mass and, assuming 


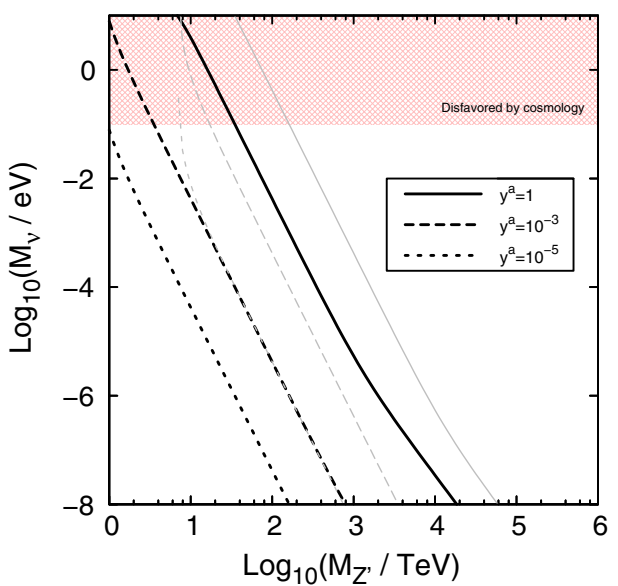

FIG. 2 (color online). Neutrino mass versus $Z^{\prime}$ scale for various values of the Dirac mass parameter $M_{D}$. Solid, dashed, and dotdashed lines correspond to $y^{a}=1,10^{-3}$, and $10^{-5}$ respectively. $g_{1}=0.6$ and $k_{2}=90 \mathrm{GeV}$, and the scale of the new colored states $\left(n_{2}\right)$ is fixed at $1 \mathrm{TeV}$ (thick lines) and $10 \mathrm{TeV}$ (thin lines).

Yukawas of order 1, would increase the exotic (primed) quark masses.

This brings us to the discussion of the quark sector. The first two generations, $Q_{L}^{1}$ and $Q_{L}^{2}$, transform as triplets of $S U(3)_{L}$, whereas the third one, $Q_{L}^{3}$, is antitriplet,

$Q_{L}^{1}=\left(\begin{array}{c}u \\ d \\ d^{\prime}\end{array}\right)_{L}, \quad Q_{L}^{2}=\left(\begin{array}{c}c \\ s \\ s^{\prime}\end{array}\right)_{L}, \quad Q_{L}^{3}=\left(\begin{array}{c}b \\ t \\ t^{\prime}\end{array}\right)_{L}$.

All right-handed states are $S U(3)_{L}$ singlets. In order to pair up the new left-handed fields of the quark sector, we introduce extra right-handed fields $d_{R}^{\prime}, s_{R}^{\prime}$, and $t_{R}^{\prime}$ that are singlets of $S U(3)_{L}$. Note that the axial anomaly cancels in this model because we have an equal number of triplets and antitriplets, and the sum of the electric charges on all the fermions vanishes [33].

The Yukawa Lagrangian of the quark sector is

$$
\begin{aligned}
\mathcal{L}_{\text {quarks }}= & y_{\alpha, i}^{u} \overline{Q_{L}^{\alpha}} \hat{u}_{R}^{i} \phi_{1}^{*}+y_{3, i}^{u} \overline{Q_{L}^{3}} \hat{u}_{R}^{i} \phi_{3} \\
& +y_{3, i}^{d} \overline{Q_{L}^{3}} \hat{d}_{R}^{i} \phi_{1}+y_{\alpha, i}^{d} \overline{Q_{L}^{\alpha}} \hat{d}_{R}^{i} \phi_{3}^{*}+\text { H.c. },
\end{aligned}
$$

$\alpha=1,2$ and $i=1, \ldots, 4(5)$ for the up (down) sector. Contractions over $i$ and $\alpha$ indices are assumed. Note that we made use of the same auxiliary parity symmetry to charge $Q_{L}^{3}$ and $\hat{d}_{R}$. After spontaneous symmetry breaking, the top mass is proportional to $k_{2}$, while the bottom mass is proportional to $k_{1}$. Hence $k_{1}$ and $k_{2}$ determine the electroweak scale. On the other hand, the masses of the extra quarks $d^{\prime}, s^{\prime}, t^{\prime}$ are proportional to $n_{1}$ and $n_{2}$, which must be of the order of $\mathrm{TeV}$ or greater in order to escape detection at the LHC. Here we note a novel feature of this model, namely, that while the extra quarks have standard electric charges, they carry two units of lepton number, relating them directly to the lepton sector.

In summary, we have proposed a new mechanism to generate neutrino mass based on the $S U(3)_{L} \otimes U(1)_{X}$ gauge symmetry. At tree level, neutrinos are massless because of lepton number conservation. Gauge interactions violate lepton number and lead to a Majorana mass term for light neutrinos at the one-loop level. In contrast to most neutrino mass generation schemes, such as the seesaw mechanism, where the neutrino mass comes from Yukawa couplings, here it arises directly from gauge boson exchange, as seen in Fig. 1 and Eq. (19). All neutrino species are massive, and their splittings and mixing parameters can be fitted to the oscillation data. The further imposition of genuine flavor symmetries would bring in predictions for oscillation parameters and possibly $0 \nu \beta \beta$, though we leave this for a separate investigation. Note that the messenger particles responsible for neutrino mass can be directly produced at the LHC: the $Z^{\prime}$ would be produced in the Drell-Yan process and provide a "portal" to access the isosinglet neutral leptons [12]. Moreover, if light enough, the new exotic colored states would also be produced at the LHC and would induce gaugemediated flavor-changing neutral currents, e.g., $b \rightarrow s \mu^{+} \mu^{-}$ [34], providing a double test. These issues will be taken up elsewhere.

\section{ACKNOWLEDGMENTS}

We thank Martin Hirsch and Renato Fonseca for useful discussions. The work of S. B. and J. V. was supported by MINECO Grant No. FPA2011-22975 and Multidark Consolider CSD2009-00064. S. M. thanks DFG Grant No. WI 2639/4-1.
[1] J. Beringer et al. (Particle Data Group), Phys. Rev. D 86, 010001 (2012).

[2] D. Forero, M. Tortola, and J. W. F. Valle, Phys. Rev. D 86, 073012 (2012).
[3] J. Schechter and J. W. F. Valle, Phys. Rev. D 22, 2227 (1980).

[4] J. Schechter and J. W. F. Valle, Phys. Rev. D 25, 2951 (1982). 
[5] P. Minkowski, Phys. Lett. 67B, 421 (1977).

[6] M. Gell-Mann, P. Ramond, and R. Slansky, Complex Spinors and Unified Theories, eConf. C790927, 315 (1979).

[7] T. Yanagida, Horizontal Symmetry and Masses of Neutrinos, eConf. C7902131, 95 (1979).

[8] R. N. Mohapatra and G. Senjanovic, Phys. Rev. Lett. 44, 912 (1980).

[9] S. M. Boucenna, S. Morisi, and J. W. F. Valle, arXiv:1404.3751.

[10] N. Cabibbo, Phys. Rev. Lett. 10, 531 (1963).

[11] M. Kobayashi and T. Maskawa, Prog. Theor. Phys. 49, 652 (1973).

[12] F. F. Deppisch, N. Desai, and J. W. F. Valle, Phys. Rev. D 89, 051302(R) (2014).

[13] J. Aguilar-Saavedra, F. Deppisch, O. Kittel, and J. Valle, Phys. Rev. D 85, 091301 (2012).

[14] S. Das, F. Deppisch, O. Kittel, and J. Valle, Phys. Rev. D 86, 055006 (2012).

[15] Y. Chikashige, R. N. Mohapatra, and R. D. Peccei, Phys. Lett. 98B, 265 (1981).

[16] J. Schechter and J. W. F. Valle, Phys. Rev. D 25, 774 (1982).

[17] G. Senjanovic and R. N. Mohapatra, Phys. Rev. D 12, 1502 (1975).

[18] M. Singer, J. Valle, and J. Schechter, Phys. Rev. D 22, 738 (1980).

[19] J. W. F. Valle and M. Singer, Phys. Rev. D 28, 540 (1983).
[20] R. Foot, H. N. Long, and T. A. Tran, Phys. Rev. D 50, R34 (1994).

[21] P. H. Frampton, Phys. Rev. Lett. 69, 2889 (1992).

[22] F. Pisano and V. Pleitez, Phys. Rev. D 46, 410 (1992).

[23] H. N. Long, Phys. Rev. D 53, 437 (1996).

[24] M. Tully and G. C. Joshi, Phys. Rev. D 64, 011301 (2001).

[25] P. V. Dong, H. T. Hung, and T. D. Tham, Phys. Rev. D 87, 115003 (2013).

[26] R. N. Mohapatra and J. W. F. Valle, Phys. Rev. D 34, 1642 (1986).

[27] J. Bernabeu, A. Santamaria, J. Vidal, A. Mendez, and J. W. F. Valle, Phys. Lett. B 187, 303 (1987).

[28] M. Dittmar, A. Santamaria, M. C. Gonzalez-Garcia, and J. W. F. Valle, Nucl. Phys. B332, 1 (1990).

[29] G. C. Branco, M. N. Rebelo, and J. W. F. Valle, Phys. Lett. B 225, 385 (1989).

[30] N. Rius and J. W. F. Valle, Phys. Lett. B 246, 249 (1990).

[31] E. Akhmedov, M. Lindner, E. Schnapka, and J. F. Valle, Phys. Lett. B 368, 270 (1996); Phys. Rev. D 53, 2752 (1996).

[32] M. Malinsky, J. C. Romao, and J. W. F. Valle, Phys. Rev. Lett. 95, 161801 (2005).

[33] H. Georgi and S. L. Glashow, Phys. Rev. D 6, 429 (1972).

[34] A. J. Buras, F. De Fazio and J. Girrbach, J. High Energy Phys. 02 (2014) 112. 